\title{
CLASSIFICATION OF THE SIMPLE MODULES OF THE QUANTUM WEYL ALGEBRA AND THE QUANTUM PLANE
}

\author{
VLADIMIR BAVULA \\ Mathematics Department, Kiev University \\ Vladimirskaya Str. 64, Kiev 252 617, Ukraine \\ E-mail: sveta@kinr.kiev.ua
}

Let $R=\oplus_{i \in \mathbf{Z}} R_{i}$ be a $\mathbf{Z}$-graded ring with a ring $R_{0}=D$, every $R_{i}=D v_{i}, v_{i} \in R_{i}$, is a left free $D$-module of rank $1, v_{0}=1$, exists a ring automorphism $\sigma$ of $D$ such that

$$
\alpha v_{i} \beta v_{j}=\alpha \sigma^{i}(\beta) c(i, j) v_{i+j}
$$

for all $\alpha, \beta \in D$ and $i, j \in \mathbf{Z}$ and some map (a "2-cocycle") $c: \mathbf{Z} \times \mathbf{Z} \rightarrow Z(D)$, the centre of $D$. Throughout the paper a "module" means a left module.

Nontrivial examples of $R$ are generalized Weyl algebras. Let $D$ be a ring, $\sigma \in$ Aut $(D)$, $a$ a central element of $D$. The generalized Weyl algebra (GWA) $A=D(\sigma, a)$ (of degree 1 ) is the ring generated by $D$ and by two indeterminates $X=v_{1}$ and $Y=v_{-1}$ subject to the relations [Bav 1]:

$$
X \alpha=\sigma(\alpha) X \text { and } Y \alpha=\sigma^{-1}(\alpha) Y, \forall \alpha \in D, Y X=a \text { and } X Y=\sigma(a) \text {. }
$$

If $D$ is commutative resp. $D=K[H]$ (the polynomial ring with coefficients in a field $K)$, the ring $A$ is also considered in [Jor 1, 2] resp. [Hod 1]. It is easy to verify that the subring of $R$ generated by $D, v_{n}, v_{-n}$ is the GWA $D\left(\sigma^{n}, c(-n, n)\right)$.

In [BVO] the simple $R$-modules were classified (up to irreducible elements of some Euclidean ring ) when $D$ is a Dedekind ring and $c(i, j) \neq 0$ for all $i, j \in \mathbf{Z}$ ( and do the same for an $\mathbf{N}$-graded ring $R$ ). As a consequence there were described all simple modules of some classical algebras. Name some of them: the first Weyl algebra $A_{1}$ and its quantum deformation $A_{1}(q)$, the universal enveloping algebra $U s l(2)$ of the Lie algebra $s l(2)$ and its quantum analog $U_{q} s l(2)$, the quantum plane $\Lambda$, the Smith's algebra [Sm], the Witten's first and Woronowicz's deformations [Za], [Bav 5]; the quantum group $\mathcal{O}_{q^{2}}(s o(k, 3))$

1991 Mathematics Subject Classification: Primary 16D10, 17B10; Secondary 16S35, 16S34.

The paper is in final form and no version of it will be published elsewhere. 
[Sm 1]; the quantum Heisenberg algebra [Ma], [KS], [Ros], [Bav 5]; and many others. Remark that all these algebras are GWA's [Bav 5,6].

The aim of this short paper is to apply the results of [BVO] and to classify the simple modules of the quantum Weyl algebra $A_{1}(q), q \neq 0,1$, and the quantum plane $\Lambda$. Moreover, we construct a great deal of (new) simple non-weight modules over the Virasoro Lie algebra. In more detail, some natural factor-algebra $V$ of the universal enveloping algebra of the Virasoro algebra is an instance of the ring $R$ as above and we classify the simple $V$-modules. The proofs are omitted since they can be found in [BVO].

The first step in the classification of simple modules of infinite dimensional algebras was made by Block [Bl 1-3]. In [Bl 3] the simple modules over the first Weyl algebra $A_{1}$ and the universal enveloping algebra $U s l(2)$ were classified (up to irreducible elements of some Euclidean ring ). Moreover, it was done for a differential operator ring $D[X ; \partial]$ with Dedekind $D$ and under some restriction on prime ideals of $D$ and on the derivation $\partial$ of $D$ (condition (3.1.1) in [Bl 3]). The simple $K[[X]][\partial]$-modules are classified in [EL]. The results of previes studies of the simple $A_{1}$-modules ([Bam], [Di 1,2]) and of $s l(2)$-modules ([AP 1,2], [Le]) are fragmentary.

The author [Bav 2-4] classified the simple modules of a generalized Weyl algebra $D(\sigma, a)$ with Dedekind $D$ and an automorphism $\sigma$ such that all maximal ideal of $D$ are linear, i.e. $\mathbf{p} \neq \sigma^{n}(\mathbf{p})$ for all $n \neq 0 \in \mathbf{Z}$ (a non-linear maximal ideal is called cyclic ). If a ground field $K$ has characteristic 0 , the Weyl algebra $A_{1}$ and all infinite dimensional prime factors of $U s l(2)$ and of $U_{q} s l(2)$ are GWA's of this kind. The quantum Weyl algebra $A_{1}(q)=<X, \partial \mid \partial X-q X \partial=1>,(q \neq 0,1 \in K)$ and the quantum plane $\Lambda=K<X, Y \mid X Y=\lambda Y X>, \lambda \in K$, are GWA's $A_{1}(q) \simeq K[H](\sigma, H), \sigma(H)=H-1$, and $\Lambda \simeq K[H](\sigma, H), \sigma(H)=\lambda H$, with $D=K[H]$, the polynomial ring (Dedekind) where exist cyclic maximal ideals (more precisely, $\left(H-(1-q)^{-1}\right)$ and $(H)$ are the unique cyclic or even $\sigma$-invariant maximal ideals of $K[H]$ respectively). It was unclear what to do in general situation (when cyclic maximal ideals exist).

Now we describe in more detail principal results of [BVO]. For a ring $A$ we denote by $\hat{A}$ the set of isomorphism classes of simple $A$-modules. Let $R$ be as above with Dedekind $D$ and $c(i, j) \neq 0$ for all $i, j \in \mathbf{Z}$. Denote by $k$ the field of fractions of $D$, i.e. $k=S^{-1} D$, $S:=D \backslash\{0\}$. Then the localization $B=S^{-1} R$ of the ring $R$ is the skew Laurent polynomial ring $B=k\left[X, X^{-1} ; \sigma\right]$ with coefficients in the field $k$ wich contains $R(R \rightarrow B$, $r \rightarrow r / 1)$. It is an Euclidean ring and, hence, a principal left and right ideal domain. By a classical theory the modules of $B$ can be described in terms of factorization of elements of $B$. We recall [Jac] that a $B$-module $M$ is simple if and only if $M \simeq B / B b$ for some irreducible $b \in B$ (i.e. $b=a c$ implies $a$ or $c$ is a unit ), and $B$-modules $B / B a$ and $B / B b$ are isomorphic if and only if $a$ and $b$ are similar, i.e. there exists $c \in B$ such that 1 is a greatest common right divisor of $b$ and $c$ and $a c$ is a least common left multiple of $b$ and $c$. If $M$ is a simple $R$-module, then the localization $S^{-1} M=B \otimes_{A} M$ of $M$ at $S$ is either 0 or a (nonzero) simple $B$-module. In accordance with these two possibilities we say that $M$ is $D$-torsion or D-torsionfree and we have the partition

$$
\hat{R}=\hat{R}(D-\text { torsion }) \cup \hat{R}(D-\text { torsionfree })
$$

(here and throughout the paper we use the following notation: if $P$ is an isomorphism- in- 
variant property of simple $R$-modules, then $\hat{R}(P)=\{[M] \in \hat{R} \mid M$ has the property $P\})$.

An $R$-module $M$ is weight, if $M$ is a semisimple $D$-module. It is easy to show that

$$
\hat{R}(D-\text { torsion })=\hat{R}(D-\text { weight }) .
$$

In the case of $\operatorname{Usl}(2, \mathbf{C})$ these weight modules are precisely the weight modules in the usual sence. So, we split our problem into two parts: the first, to describe $\hat{R}(D$ - weight $)$ and the second, $\hat{R}(D$-torsionfree). The first is much more simpler (although there are a lot of types of simple weight modules) and much more "technical". This is the reason why we say nothing here about these modules. The first part is more "geometrical", the second - more "arithmetical".

An $R$-module $M$ is called $R$-socle if the socle $\operatorname{Soc}_{R}(M)$ (= sum of the simple $R$ submodules of $M)$ is nonzero. Denote by $\hat{B}(R$ - socle $)$ the set of isoclasses of simple $B$-modules which are $R$-socle. An element $b \in B$ is $R$-socle if $B / B b$ is so. Lemma 5.1, [BVO], gives a describtion of simple $D$-torsionfree $R$-modules in terms of simple $R$-socle $B$-modules and gives a form of any simple $D$-torsionfree $R$-module.

- (Lemma 5.1, [BVO]) The canonical map

$$
S^{-1}: \hat{R}(D-\text { torsionfree }) \rightarrow \hat{B}(R \text {-socle }),[M] \rightarrow\left[S^{-1} M\right],
$$

is bijective with inverse Soc $:[N] \rightarrow\left[\operatorname{Soc}_{R}(N)\right]$. Each simple D-torsionfree $R$ module has the form $M_{\mathbf{m}}:=R / R \cap B \mathbf{m}$ for some left maximal ideal $\mathbf{m}$ of $B$ and $M_{(\mathbf{m}=B b)} \simeq M_{(\mathbf{n}=\mathbf{B c})}$ are isomorphic if and only if $B / \mathbf{m} \simeq B / \mathbf{n}$ as B-modules, i.e. the corresponding irreducible elements $b$ and $c$ are similar.

If $R$ is a GWA $D(\sigma ; a \neq 0)$ and all maximal ideals of $D$ are linear, then $\hat{B}(R-$ socle $)=$ $\hat{B}=k\left[X, X^{-1} ; \sigma\right]^{\wedge}$, [Bav 2-4]. In general, it is not the case, moreover, in "most" cases simple $B$-modules are not $R$-socle (Section 6, [BVO]). The following result illustrates these words:

- (Theorem 5.14, $[\mathrm{BVO}]) \hat{R}(D$ - torsionfree $) \neq \emptyset$ if and only if there are no more than finitely many cyclic maximal ideals of $D$.

It means that so less $D$ has cyclic maximal ideals so much the ring $R$ has the simple modules.

The cyclic group $G$, generated by $\sigma$, acts in an obvious way on the set $\operatorname{Specm}(D)$ of maximal ideals of $D$. An orbit $\mathcal{O}(\mathbf{p})=\left\{\sigma^{i}(\mathbf{p}), i \in \mathbf{Z}\right\}$ of a maximal ideal $\mathbf{p}$ is linear resp. cyclic if $\mathbf{p}$ is so. Let $\alpha, \beta \in S$, we write $\alpha<\beta$ if there are no maximal ideals $\mathbf{p}$ and $\mathbf{q}$ of $D$ which belong to the same linear orbit and such that $\alpha \in \mathbf{p}, \beta \in \mathbf{q}$ and $\mathbf{p}=\sigma^{i}(\mathbf{q})$ for some $i \geq 0$. An element $b=v_{-m} \beta_{-m}+\cdots+\beta_{0} \in R$, all $\beta_{i} \in D, \beta_{-m} \neq 0, \beta_{0} \neq 0$, $m>0$, is called $l$-normal if $\beta_{0}<\beta_{-m}$, and $\beta_{0}<a:=c(-1,1)$.

Let $\mathcal{O}(\mathbf{p})$ be a cyclic orbit which contains $n$ elements. Set $\theta(\mathcal{O})$ for $\sqcap_{i=0}^{n-1} \sigma^{i}(\mathbf{p})$, the product of all ideals from $\mathcal{O}$. Theorem 5.13, [BVO], gives a necessary and sufficient condition for a module $R / R \cap B b$ to be $R$-socle, where $b$ is irreducible in $B$ :

- (Theorem 5.13, [BVO]) $R / R \cap B b$ is $R$-socle if and only if (CO) holds :

(CO) $\quad R=R \theta(\mathcal{O})+R \cap B b$ for all cyclic orbit $\mathcal{O}$.

Moreover, if $b$ is l-normal, then the $R$-module $R / R \cap B b$ is simple. 
Remark that (CO) is to be verified only for finitely many orbits (see Theorem 5.14 above). To sum up Lemma 5.1 and Theorem 5.13 we have.

Theorem A ([BVO]). Let $R$ be as above with Dedekind $D$ and $c(i, j) \neq 0$ for all $i, j \in \mathbf{Z}$, and $b$ be l-normal irreducible in $B$ and $(\mathbf{C O})$ holds. Then $R / R \cap B b$ is a $D$ torsionfree simple $R$-module $\left(=\operatorname{Soc}_{R}(B / B b)\right)$. Up to isomorphism every $D$-torsionfree simple $R$-module arises in this way, and from $a b$ which is unique up to similarity.

- $($ Proposition 5.16, $[\mathrm{BVO}])$ If $\mathcal{O}(\mathbf{p})=\{\mathbf{p}\}$, i.e. $\mathbf{p}=\sigma(\mathbf{p}), c(-1,1) \notin \mathbf{p}, b \notin R \mathbf{p}$, then (CO) holds for the orbit $\mathcal{O}$ if and only if exists exactly one $\beta_{i}$ th such that $\beta_{i} \notin \mathbf{p}$.

In Section 1 we apply these results to classify the simple modules over the quantum Weyl algebra $A_{1}(q), q \neq 0,1$, over an algebraically closed field $K$. If char $K=0$, then for $A_{1}(q)$ there exists a unique cyclic maximal ideal, it is $\sigma$-invariant and satisfies the assumption of Proposition 5.16. On the contrary, in the case of the Weyl algebra $A_{1}$ there are no cyclic ideals at all. We see that existence of only one cyclic ideal in the case of $A_{1}(q)$ reduces substantially the set of simple modules in comparison with $A_{1}$.

1. The simple modules of the quantum Weyl algebra. Let $K$ be a field algebraically closed for simplicity.

The quantum Weyl algebra $A_{1}(q)=<X, \partial \mid \partial X-q X \partial=1>$ over $K(q \neq 0,1 \in K)$ is the GWA :

$$
A_{1}(q) \simeq K[H](\sigma, a=H), X \leftrightarrow X, \partial \leftrightarrow Y, \partial X \leftrightarrow H,
$$

with $\sigma: \sigma(H)=q^{-1}(H-1)$.

Identify Specm $K[H]$ with $K$ by the map $(H-\lambda) \rightarrow \lambda$. Then $\sigma$ "acts" on $K$ as $\sigma(\lambda)=q \lambda+1$. Therefore, any orbit has the form $\mathcal{O}(\lambda)=\left\{\sigma^{i}(\lambda)=q^{i} \lambda+\left(q^{i}-1\right) /(q-1)\right.$, $i \in \mathbf{Z}\}$, the element $\delta:=(1-q)^{-1}$ is $\sigma$-invariant, i.e. $\mathcal{O}(\delta)=\{\delta\}$.

$q$ is an n'th root of $1\left(q^{n}=1\right)$. In this case $\sigma^{n}=1$, hence all orbits are cyclic, by Theorem 5.14 (from Introduction) each simple $A_{1}(q)$-module is $K[H]$-torsion $\equiv$ weight.

All orbits but $\mathcal{O}(\delta)$ are of length $n, \mathcal{O}(0)$ is the unique degenerate orbit.

Corollary 1.1. If $q$ is an $n$ 'th root of 1 . Then each simple $A_{1}(q)$-module is finite dimensional and weight and the set

$$
\hat{A}_{1}(q)=\hat{A}_{1}(q)( \pm \mathbf{0}) \cup \hat{A}_{1}(q)(-,-) \cup \hat{A}_{1}(q)(+,+) \cup \hat{A}_{1}(q)(-,+) \text { (a disjoint union) }
$$

is described by (1.1)-(1.4) below. .

$\hat{A}_{1}(q)( \pm \mathbf{0})$ contains the unique class which corresponds to the module

$$
L( \pm \mathbf{0})=A_{1}(q) / A_{1}(q)\left(X, H, Y^{n}\right) .
$$

For $\mathbf{t}=(-,-),(+,+)$, the map

$$
K^{*}:=K \backslash\{0\} \rightarrow \hat{A}_{1}(q)(\mathbf{t}), \lambda \rightarrow[L(\mathbf{t}, \lambda)],
$$

is bijective, where

$$
\begin{gathered}
L((-,-), \lambda)=A_{1}(q) / A_{1}(q)\left(X^{n}, H, Y^{n}-\lambda\right) \text { and } \\
L((+,+), \lambda)=A_{1}(q) / A_{1}(q)\left(Y^{n}, H, X^{n}-\lambda\right) .
\end{gathered}
$$


The map

$$
K^{*} \times K^{*} \cup K^{*} \rightarrow \hat{A}_{1}(q)(-,+),(\lambda, \mu) \rightarrow[L((-,+), \lambda, \mu)], \mu \rightarrow[L((-,+), \mu)],
$$

is bijective, where

$$
\begin{gathered}
L((-,+), \lambda, \mu)=A_{1}(q) / A_{1}(q)\left(H-\lambda, X^{n}-\mu,\right. \\
\left.Y^{n}-\mu^{-1} \lambda(q \lambda+1) \cdots\left(q^{n-1} \lambda+\left(q^{n}-1\right) /(q-1)\right)\right)
\end{gathered}
$$

and

$$
L((-,+), \mu)=A_{1}(q) / A_{1}(q)\left(H-\delta, X-\mu, Y-\delta \mu^{-1}\right) .
$$

$q$ is not a root of $1 . \hat{A}_{1}(q)$ (weight). In this case there is the unique cyclic orbit $\mathcal{O}(\delta)$, $\delta=(1-q)^{-1}$, which is nondegenerate.

Corollary 1.2. If $q$ is not a root of 1 , then

$$
\hat{A}_{1}(q)(\text { weight })=\hat{A}_{1}(q)(\text { weight, linear }) \sqcup \hat{A}_{1}(q)(-,+)
$$

(a disjoint union) is described by (1.5) and (1.6).

Two scalars $\lambda$ and $\mu$ from $K \backslash \delta$ are equivalent iff $\lambda, \mu$ belong to $\mathcal{O} \neq \mathcal{O}(0)$ or to $\Gamma_{1}:=\left\{\sigma^{i}(0), i \leq 0\right\}$ or to $\Gamma_{2}:=\left\{\sigma^{i}(0), i>0\right\}$.

The following map is bijective:

$$
\begin{gathered}
(K \backslash \delta) / \sim \rightarrow \hat{A}_{1}(q) \text { (weight, linear), } \mathcal{O}(\lambda \neq 0) \rightarrow\left[L(\mathcal{O}(\lambda))=A_{1}(q) / A_{1}(q)(H-\lambda)\right] \\
\Gamma_{1} \rightarrow\left[L\left(\Gamma_{1}\right)=A_{1}(q) / A_{1}(q)(H, X)\right], \quad \Gamma_{2} \rightarrow\left[L\left(\Gamma_{2}\right)=A_{1}(q) / A_{1}(q)(H-1, Y)\right] .
\end{gathered}
$$

The map

$$
K^{*} \rightarrow \hat{A}_{1}(q)(-,+), \mu \rightarrow\left[L((-,+), \lambda)=A_{1}(q) / A_{1}(q)\left(H-\delta, X-\mu, Y-\mu^{-1} \delta\right)\right],
$$

is bijective. It is clear that each module in (1.6) is 1-dimensional.

$\hat{A}_{1}(q)$ (D-torsionfree), $D=K[H]$. The localization $B$ of $A_{1}(q)$ at $S=K[H] \backslash\{0\}$ is the skew Laurent polynomial ring:

$$
B=K(H)\left[X, X^{-1} ; \sigma\right], \sigma(H)=q^{-1}(H-1),
$$

with coefficients in the field $K(H)$ of rational functions.

An element $f=v_{-m} \beta_{-m}+\cdots+\beta_{0} \in A_{1}(q)$ of length $m>0$, all $\beta_{i} \in K[H]$, is $l$-normal if and only if the following condition holds:

$\left(l\right.$-norm): every scalar $\sigma^{i}(0)=\left(q^{i}-1\right) /(q-1), i \geq 0$, is not a root of $\beta_{0}$ (i.e. $\beta_{0}<$ $a=H)$ and if $\lambda$ and $\mu=\sigma^{j}(\lambda)=q^{j} \lambda+\left(q^{j}-1\right) /(q-1)$ are roots of polynomials $\beta_{0}$ and $\beta_{-m}$ respectively, then $j>0$ (i.e. $\beta_{0}<\beta_{-m}$ ).

Corollary 1.3. Let $q$ be not a root of 1 and $M$ be a simple $K[H]$-torsionfree $A_{1}(q)$ module; then $M \simeq A_{1}(q) / A_{1}(q) \cap B f$ for an irreducible element $f=v_{-m} \beta_{-m}+\cdots+\beta_{0}$ of $B$ (all $\beta_{i} \in K[H], \beta_{0} \neq 0, \beta_{-m} \neq 0, m>0$ ) such that (l-norm) holds and all polynomials $\beta_{i}$ but exactly one have the root $(1-q)^{-1}$. Two of these $A_{1}(q)$-modules being isomorphic if and only if the corresponding $f$ are similar in $B$.

Re mark. Any scalar is a root of 0 . 
2. Simple modules of the Virasoro algebra. Let $K$ be an algebraically closed field of characteristic zero.

The first Weyl algebra $A_{1}=K<X, \partial \mid \partial X-X \partial=1>$ is isomorphic to the GWA:

$$
A_{1} \simeq K[H](\sigma, a=H), \sigma(H)=H-1, X \leftrightarrow X, \partial \leftrightarrow Y, \partial X \leftrightarrow H .
$$

Let $V i r$ be the Virasoro Lie algebra, i.e. the infinite dimensional vector space with basis $e_{i}, i \in \mathbf{Z}, c$, where the Lie algebra structure is defined by

$$
\left[e_{i}, e_{j}\right]=(j-i) e_{i+j}+\left(j^{3}-j\right) \delta_{i,-j} c / 12
$$

and $c$ is the central element of $V i r$. Denote by $V$ the image of the algebra homomorphism from the universal enveloping algebra $U(V i r)$ to the localization $A_{1,(X)}$ of the first Weyl algebra $A_{1}$ at the multiplicatively closed subset $\left\{X^{i}, i \geq 0\right\}$ and defined as

$$
U(V i r) \rightarrow V \subset A_{1,(X)}, e_{i} \rightarrow X^{i+1} \partial, c \rightarrow 0 .
$$

$V$ is a homogeneous subalgebra of the $\mathbf{Z}$-graded algebra (the skew Laurent polynomial ring):

$$
A_{1,(X)}=K[H](\sigma, H)_{(X)}=K[H]\left[X, X^{-1} ; \sigma\right], \sigma(H)=H-1,
$$

Thus $V$ is the example of the $\operatorname{ring} R$ with $D=K[H]$, the polynomial ring, and the automorphism $\sigma: H \rightarrow H-1$. In fact, setting $v_{i}=X^{i+1} \partial, i \neq 0 \in \mathbf{Z}, v_{0}=1, H=X \partial+1$, we see that

is $\mathbf{Z}$-graded with

$$
V=\oplus_{i \in \mathbf{Z}} D v_{i}
$$

$$
\begin{gathered}
v_{i} v_{j}=(H-i-1) v_{i+j}, \text { if } i+j \neq 0, \\
\left(v_{i} v_{j}=X^{i+1} \partial X^{j+1} \partial=X^{i} \sigma(H) X^{j+1} \partial=\sigma^{i+1}(H) X^{i+j+1} \partial=\sigma^{i+1}(H) v_{i+j}\right), \text { and } \\
v_{-i} v_{i}=(H+i-1)(H-1), \text { if } i \neq 0 \in \mathbf{Z} .
\end{gathered}
$$

Moreover, $V$ is affine and generated over $K$ by $H, v_{ \pm 1}$ and $v_{ \pm 2}$.

We shall describe $\hat{V}$.

The automorphism $\sigma$ "acts" on the set of maximal ideals $K \equiv \operatorname{Specm} D(\lambda \rightarrow(H-\lambda)$, where $D=K[H])$ as $\sigma: \lambda \rightarrow \lambda+1$. Thus an orbit equals to $\lambda+\mathbf{Z}$ for some $\lambda \in K$.

Define on $K$ an equivalence relation $\sim$ as follows: $\lambda \sim \mu$ if both $\lambda$ and $\mu$ belong to $\Gamma_{1}=\{1\}$ or to $\Gamma_{2}=\mathbf{Z} \backslash\{1\}$ or to an orbit $\gamma+\mathbf{Z} \neq \mathbf{Z}$.

Corollary 2.1. The map

$$
K / \sim \hat{V}(\text { weight }), \Gamma \rightarrow[L(\Gamma)],
$$

is bijective with inverse $[L] \rightarrow$ Supp L, where

1. $\Gamma_{1}=\{1\}, L\left(\Gamma_{1}\right)=V /\left(D(H-1)+\sum_{i \neq 0 \in \mathbf{Z}} D v_{i}\right) \simeq K$;

2. $\Gamma_{2}=\mathbf{Z} \backslash\{1\}, L\left(\Gamma_{2}\right)=V / D\left(H, v_{1}\right)$;

3. $\Gamma=\lambda+\mathbf{Z}(\lambda \notin \mathbf{Z}), L(\Gamma)=V / V(H-\lambda)$.

The localization $B=S^{-1} V$ of $V$ at $S=D \backslash\{0\}$ is the skew Laurent polynomial ring

$$
B=K(H)\left[X, X^{-1} ; \sigma\right], \sigma(H)=H-1,
$$

with coefficients in the field $K(H)$ of rational functions. 
Corollary 2.2. Let $M$ be a simple $K[H]$-torsionfree $V$-module, then $M \simeq V / V \cap B f$ for an irreducible element $f=v_{-m} \beta_{-m}+\cdots+\beta_{0}$ of $B$ (all $\beta_{i} \in K[H], \beta_{0} \neq 0, \beta_{-m} \neq 0$, $m>0)$ such that all roots of $\beta_{0}$ and all the differences $\lambda-\mu$ of the roots $\lambda$ of $\beta_{0}$ and $\mu$ of $\beta_{-m}$ are not non-negative integers. Two of these $V$-modules being isomorphic if and only if the corresponding $f$ are similar in $B$.

For a polynomial $\alpha \in K[H]$ set $[\alpha]:=\cup\{\lambda+\mathbf{Z}\}$ where $\lambda$ runs through all roots of $\alpha$.

Lemma 2.3. Let $p=\alpha v_{1}+\beta$ or $p=\alpha v_{-1}+\beta$ where $\alpha \neq 0, \beta \neq 0 \in K[H]$ be such that $\beta$ has no roots from $\mathbf{Z}$ and $[\alpha] \cap[\beta]=\emptyset$. Then $V / V p$ is a simple $K[H]$-torsionfree $V$-module.

The case of char $K=p>0$ is left to the reader. Since $\sigma^{p}=1$, by Theorem 5.14 every simple $V$-module is weight and finite dimensional and the set $\hat{V}$ can be easily described.

3. The simple modules of the quantum plane. The quantum plane

$$
\Lambda=K<X, Y \mid X Y=q Y X>, q \neq 0 \in K,
$$

is isomorphic to the generalized Weyl algebra:

$$
\Lambda \simeq K[H](\sigma, a=H), X \leftrightarrow X, Y \leftrightarrow Y, Y X \leftrightarrow H, \sigma(H)=q H .
$$

Identify Specm $K[H]$ with $K$ by the map $(H-\lambda) \rightarrow \lambda$. Then $\sigma$ "acts" on $K$ as $\sigma(\lambda)=q^{-1} \lambda$. Therefore, any orbit has the form $\mathcal{O}(\lambda)=\left\{\sigma^{i}(\lambda)=q^{-i} \lambda, i \in \mathbf{Z}\right\}$. The element 0 is $\sigma$-invariant, i.e. $\mathcal{O}(0)=\{0\}$.

$q$ is an $n^{\text {th }}$ root of $1\left(q^{n}=1\right)$. In this case $\sigma^{n}=1$, hence all orbits are cyclic, by Theorem 5.14 (see Introduction) each simple $\Lambda$-module is $K[H]$-torsion $\equiv$ weight, moreover, it is finite dimensional.

All orbits but $\mathcal{O}(0)$ are of length $n, \mathcal{O}(0)$ contains the only one element and is the unique degenerate orbit.

COROllary 3.1. If $q$ is an $n$ 'th root of 1 . Then each simple $\Lambda$-module is finite dimensional and weight and the set

$$
\hat{\Lambda}=\hat{\Lambda}( \pm \mathbf{0}) \cup \hat{\Lambda}(-,-) \cup \hat{\Lambda}(+,+) \cup \hat{\Lambda}(-,+)
$$

(a disjoint union) is described by (3.1)-(3.3).

$\hat{\Lambda}( \pm \mathbf{0})$ contains the unique class which corresponds to the module

$$
L( \pm \mathbf{0})=\Lambda / \Lambda(X, H, Y) .
$$

For $\mathbf{t}=(-,-),(+,+)$, the map

$$
K^{*}:=K \backslash\{0\} \rightarrow \hat{\Lambda}(\mathbf{t}), \lambda \rightarrow[L(\mathbf{t}, \lambda)],
$$

is bijective, where

$$
L((-,-), \lambda)=\Lambda / \Lambda(X, H, Y-\lambda) \text { and } L((+,+), \lambda)=\Lambda / \Lambda(Y, H, X-\lambda) .
$$

The map

$$
K^{*} \times K^{*} \rightarrow \hat{\Lambda}(-,+),(\lambda, \mu) \rightarrow[L((-,+), \lambda, \mu)],
$$

is bijective, where

$$
L((-,+), \lambda, \mu)=\Lambda / \Lambda\left(H-\lambda, X^{n}-\mu, Y^{n}-\mu^{-1} \lambda^{n} q^{n(n-1) / 2}\right) .
$$


$q$ is not a root of $1 . \hat{\Lambda}$ (weight). There is the unique cyclic orbit $\mathcal{O}(0)$, it is the unique degenerate orbit. Thus

$$
\hat{\Lambda} \text { (weight })=\hat{\Lambda}(\text { weight, linear }) \sqcup \hat{\Lambda}( \pm \mathbf{0}) \sqcup \hat{\Lambda}((-,-)) \sqcup \hat{\Lambda}((+,+)) \text {. }
$$

The last three types of simple $\Lambda$-modules $(\hat{\Lambda}( \pm \mathbf{0}), \ldots)$ are described by (3.1) and (3.2).

Specm.lin $K[H]=K \backslash 0$ and two scalalrs $\lambda$ and $\mu$ from $K \backslash 0$ are equivalent if and only if $\lambda=\sigma^{i}(\mu)=q^{-i} \mu$ for some $i \in \mathbf{Z}$, if and only if they belong to the same orbit.

The map

$$
(K \backslash 0) / \sim \rightarrow \hat{\Lambda} \text { (weight, linear), } \mathcal{O}(\lambda) \rightarrow[L(\mathcal{O}(\lambda))=\Lambda / \Lambda(H-\lambda)]
$$

is bijective.

Corollary 3.2. If $q$ is not a root of 1 , then $\hat{\Lambda}$ (weight) is described by (3.4) and (3.1), (3.2), (3.5).

$\hat{\Lambda}(D$-torsionfree $), D=K[H]$.

The localization $B$ of $\Lambda$ at the multiplicatively closed set $S=K[H] \backslash\{0\}$ is the skew Laurent polynomial ring:

$$
B=K(H)\left[X, X^{-1} ; \sigma\right], \sigma(H)=q H,
$$

with coefficients in the field $K(H)$ of rational functions.

An element $f=v_{-m} \beta_{-m}+\cdots+\beta_{0} \in \Lambda$ of length $m>0$, all $\beta_{i} \in K[H]$, is l-normal if and only if the following condition holds:

( $l$-norm): If $\lambda$ and $\mu=\sigma^{j}(\lambda)=q^{-j} \lambda$ are roots of polynomials $\beta_{0}$ and $\beta_{-m}$ respectively, then $j>0$ (i.e. $\beta_{0}<\beta_{-m}$ ).

Remark that $\beta_{0}<a=H$ since $(a=H)$ is a cyclic orbit.

Corollary 3.3. Let $q$ be not a root of 1 and $M$ be a simple $K[H]$-torsionfree $\Lambda$ module, then $M \simeq \Lambda / \Lambda \cap B f$ for an irreducible element $f=v_{-m} \beta_{-m}+\cdots+\beta_{0}$ of $B$ (all $\beta_{i} \in K[H], \beta_{0} \neq 0, \beta_{-m} \neq 0, m>0$ ) such that (l-norm) holds and $\Lambda=\Lambda H+\Lambda \cap B f$. Two of these $\Lambda$-modules being isomorphic if and only if the corresponding $f$ are similar in $B$.

\section{References}

[AP 1] D. Arnal and G. Pinczon, On algebraically irreducible representations of the Lie algebra $s l(2)$, J. Math. Phys. 15 (1974), 350-359.

[AP 2] D. Arnal and G. Pinczon, Idéaux à gauche dans les quotients simples de l'algèbre enveloppante de $s l(2)$, Bull. Soc. Math. France 101 (1973), 381-395.

[Bam] K. S. Bamba, Sur les idéaux maximaux de l'algèbre de Weyl $A_{1}$, C. R. Acad. Sci. Paris (A) 283 (1976), 71-74.

[BVO] V. V. Bavula, and F. van Oystaeyen, The simple modules of certain generalized crossed products, Trans. Amer. Math. Soc. (to appear).

[Bav 1] V. V. Bavula, The finite-dimensionality of Ext ${ }^{n}$ 's and Tor $_{n}$ 's of simple modules over a class of algebras, Funktsional. Anal. i Prilozhen. 25 (1991),no. 3, 80-82.

[Bav 2] V. V. Bavula, The simple $D[X, Y ; \sigma, a]$-modules, Ukrainian Math. J. 44 (1992), 16281644 . 
[Bav 3] V. V. Bavula, Generalized Weyl algebras, kernel and tensor-simple algebras, their simple modules, Representations of algebras. Sixth International Conference, August 19-22, 1992. CMS Conference proceedings (V. Dlab and H. Lenzing Eds.), v. 14 (1993), 83-106.

[Bav 4] V. V. Bavula, Generalized Weyl algebras and their representations, Algebra i Analiz, 4 (1992), no. 1, 75-97; English trans. in St.Petersburg Math. J. 4 (1993), no. 1, 71-92.

[Bav 5] V. V. Bavula, Tensor homological minimal algebras, global dimension of the tensor product of algebras and of generalized Weyl algebras, Bull. Sci. Math. 120 (1996), 293-335.

[Bav 6] V. V. Bavula, Global dimension of generalized Weyl algebras. Proceedings of the 7th Int. Conf. on Represent. of Algebras, August 22-26, 1994. CMS Conference proceedings (R. Bautista, R. Martinez-Villa and J. A. de la Pena Eds), 18 (1996), 81-107.

[Bl 1] R. E. Block, Classification of the irréducible representations of $s l(2, \mathbf{C})$, Bull. Amer. Math. Soc., 1 (1979), 247-250. sl(2) and of the Weyl algebra, Adv. Math. 39 (1981), 69-110.

[Bl 2] R. E. Block, The irreducible representations of the Weyl algebra $A_{1}$, in "Séminaire d'Algèbre Paul Dubreil (Proceedings, Paris 1977-1978)" (M. P. Malliavin, Ed.), Lecture Notes in Mathematics no. 740, pp. 69-79, Springer-Verlag, Berlin/New York, 1979.

[Bl 3] R. E. Block, The irreducible representations of the Lie algebra $s l(2)$ and of the Weyl algebra, Adv. Math. 39 (1981), 69-110.

[Di 1] J. Dixmier, Représentations irréductibles des algèbres de Lie nilpotentes, An. Acad. Brasil. Ci. 35 (1963), 491-519.

[Di 2] J. Dixmier, Sur les algèbres de Weyl, Bull. Soc. Math. France 96 (1968), 209-242.

[EL] A. Van den Essen and A. Levelt, An explicit description of all simple $K[[X]][\partial]-$ modules, Contemp. Math., 130 (1992), 121-131.

[Jac] N. Jacobson, The Theory of Rings, Amer. Math. Soc., Providence, R. I., 1943.

[Jor 1] D. A. Jordan, Krull and global dimension of certain iterated skew polynomial rings, Contemp. Math., 130 (1992), 201-213.

[Jor 2] D. A. Jordan, Primitivity in skew Laurent polynomial rings and related rings, Math. Z., 213 (1993), 353-371.

[Hod 1] T. J. Hodges, Noncommutative deformation of type-A Kleinian singularities, J. Algebra 161 (1993), no. 2, 271-290.

[Le] F. W. Lemire, Existence of weight space decompositions for irreducible representations of simple Lie algebras, Canad. Math. Bull. 14 (1971), 113-115.

[Ma] M.-P. Malliavin, L'algèbre d'Heisenberg quantique, C. R. Acad. Sci. Paris, Sér. 1, 317 (1993), 1099-1102.

[MR] J. C. McConnell and J. C. Robson, Homomorphism and extensions of modules over certain polynomial rings, J. Algebra 26 (1973), 319-342.

[Sm] S. P. Smith, A class of algebras similar to the enveloping algebra of $s l(2)$, Trans. Amer. Math. Soc. 322 (1990), 285-314.

[Sm 1] S. P. Smith, Quantum qroups: An introduction and survey for ring theoretists, in Noncommutative Rings (S.Montgomery and L.W.Small, Eds.) pp. 131-178, MSRI publ. 24, Springer-Verlag, Berlin (1992).

[Za] C. Zachos, Elementary paradigms of quantum algebras, Contemporary Math. 134 (1992), 351-377. 